\title{
Extension de l'aire de distribution de la Couleuvre à collier, Diadophis punctatus edwardsii, dans l'est du Québec
}

\author{
JEAN-François DesRoches ${ }^{1}$ et BenOIT Roussel ${ }^{2}$ \\ ${ }^{1}$ Cégep de Sherbrooke, Département des Techniques d'écologie appliquée, 475 du Parc, Sherbrooke, Québec J1K 4K1, \\ Canada \\ ${ }^{2}$ Parc national du Saguenay, 91 Notre-Dame, Rivière-Éternité, Québec G0V 1P0 Canada
}

\begin{abstract}
Desroches, Jean-François, et Benoit Roussel. 2005. Extension de l'aire de distribution de la Couleuvre à collier, Diadophis punctatus edwardsii, dans l'est du Québec. Canadian Field-Naturalist 119(3): 457-458.
\end{abstract}

Deux Couleuvres à collier adulte (Diadophis punctatus edwardsii) ont été trouvées au parc national du Saguenay, dans l'est du Québec, en 2004 et 2005. Ces mentions se retrouvent à près 150 kilomètres au nord-nord-est de la mention la plus proche de l'espèce. L'habitat est constitué de forêt mixte près d'escarpements rocheux, dans des vallées. Il pourrait s'agir de populations isolées de celles retrouvées plus au sud.

Two Ringneck Snakes (Diadophis punctatus edwardsii) was found in the Saguenay National Park, in eastern Québec, in 2004 and 2005. These records are about 150 kilometers north-north-east from the nearest previous record. The habitat is mixed forest, with rocky cliffs, in valleys. They may represent isolated populations from those found in southern Quebec.

Mots-Clés: Couleuvre à collier, Diadophis punctatus edwardsii, aire de distribution, population isolée, habitat, Québec.

La Couleuvre à collier (Diadophis punctatus edwardsii) est une espèce discrète qui a historiquement été peu rapportée au Québec. Jusqu'en 1990 elle y était la couleuvre la moins souvent observée, les mentions de l'espèce étant confinées au sud de la province (Bider et Matte 1991). Il s'agit d'une espèce discrète et aux mœurs fouisseuses, qui estive durant les périodes chaudes et sèches (Ernst et Ernst 2003). Bien que difficile à apercevoir, l'espèce peut être abondante par endroits (Cook 1984; Ernst et Ernst 2003) et pourrait être plus répandue qu'on le croit (Desroches et Rodrigue 2004). Au Québec, la mention de Couleuvre à collier la plus à l'est, sur la côte nord du fleuve SaintLaurent, a été faite à Saint-Joachim près du Cap-Tourmente (Provancher 1874), à quelques 40 kilomètres au nord-est de la ville de Québec.

Le 22 juillet 2004, un spécimen adulte a été découvert dans le parc national du Saguenay, municipalité de Rivière-Éternité, canton de Hébert $\left(48^{\circ} 17^{\prime} 35^{\prime \prime} \mathrm{N}\right.$; $\left.70^{\circ} 20^{\prime} 38^{\prime \prime}\right)$. Sa longueur totale est d'environ $27,5 \mathrm{~cm}$. Il porte sous le ventre une série de points noirs, au centre de plusieurs des écailles ventrales. La couleuvre a été trouvée morte en bordure d'un sentier, et son état laissait présager qu'une faucheuse l'avait découpée en morceaux lors de travaux d'entretien. Le spécimen est conservé dans la collection herpétologique du Musée canadien de la nature (CMNAR-35820). L'habitat est une forêt mixte dans une vallée encaissée, où poussent le Sapin baumier (Abies balsamea), le Bouleau jaune (Betula alleghaniensis), l'Érable à sucre (Acer saccharum), le Frêne noir (Fraxinus nigra) et des épinettes (Picea sp.). La strate arbustive se compose, outre les jeunes arbres des espèces cités précédemment, de l'Érable à épis (Acer spicatum), de l'If du Canada (Taxus canadensis) et du Noisetier à long bec (Corylus cornuta).

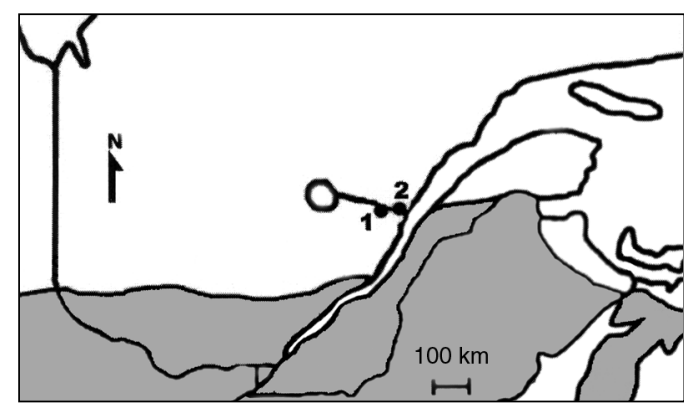

FIGURE 1. Répartition de la Couleuvre à collier au Québec et sur le territoire adjacent. Les mentions faites au parc du Saguenay sont répresentées par les points noirs numérotés $(1=$ Rivière-Éternité; $2=$ Sacré-Cœur $)$. (carte modifiée d'après Cook 1984; Bider et Matte 1994; Desroches et Rodrigue 2004).

Le 27 août 2005, un second spécimen de Couleuvre à collier adulte a été découvert dans le parc du Saguenay, municipalité de Sacré-Cœur, canton d'Albert (48 $\left.15^{\prime} 53^{\prime \prime} \mathrm{N} ; 6^{\circ} 56^{\prime} 49^{\prime \prime} \mathrm{O}\right)$. D'une longueur totale d'environ $34,0 \mathrm{~cm}$, il ne porte aucun point noir au centre des écailles ventrales. Comme ce fut le case en 2004, cette couleuvre était morte lors de sa découverte. Elle gisait sur une route d'asphalte, probablement écrasée par une automobile. Le spécimen a été récolté et déposé au Musée canadien de la nature (CMNAR-35821). L'habitat à cet endroit est une forêt mixte, où l'on retrouve l'Épinette noire (Picea mariana), le Sapin baumier, des peupliers (Populus sp.), le Bouleau blanc (Betula papyrifera), le Pin rouge (Pinus resinosa) et le Pin gris (Pinus banksiana). Beaucoup d'épines de conifères jonchent le sol. 
Ces deux mentions de Couleuvres à collier se retrouvent respectivement à 140 kilomètres et 148 kilomètres au nord-nord-est de la mention connue la plus proche de l'expèce (Saint-Joachim (Provancher 1874)) (figure 1). Elles ont été faites de part et d'autre de la rivière Saguenay, soit à l'ouest (Rivière-Éternité) et à l'est (Sacré-Cœur) et sont séparées entre elles de 30 kilomètres. En effet, la répartition de la Couleuvre à collier, dans le sud du Québec, est associée aux différents domaines de l'érablière et à celui de la sapinière à Bouleau jaune. La saison de croissance y dure de 160 à 190 jours (Robitaille et Saucier 1998). La présente mentions faites dans le parc national du Saguenay, se situe dans le domaine de la sapinière à Bouleau jaune, et la saison de croissance dure de 140 à 170 jours (Robitaille et Saucier 1998). L'Érable à sucre, et dans une moindre mesure le Bouleau jaune et le Frêne noir, poussent dans le sud du Québec et de manière isolée au Saguenay (voir les cartes présentées dans Farrar 1996). La répartition de la Couleuvre à collier pourrait être semblable.

Le territoire séparant le Saguenay de la région de Québec (mention de Provancher 1874) est très différent. Il est constitué de hautes collines et de monts, et fait partie de la zone des sapinières à Bouleau blanc, à Épinette blanche (Picea glauca), à Épinette noire et de la pessière à mousse. La saison de croissance dure de 130 à 160 jours (Robitaille et Saucier 1998). Ce territoire semble peu propice à l'établissement de la Couleuvre à collier. Nos recherches effectuées dans ce secteur ne nous ont permis d'y recenser que la Couleuvre rayée (Thamnophis sirtalis), laquelle se retrouve au Québec presque jusqu'à la latitude $54^{\circ}$ nord (MacCulloch et Bider 1975; Bider et Matte 1994; J.-F. Desroches, données non publiées).

La présence de la Couleuvre à collier au Saguenay est très intéressante; il s'agit d'une extension d'aire importante vers le nord-est pour l'espèce, et possiblement d'une population isolée. Les populations isolées de certains reptiles revêtent une importance particulière en conservation (voir dans Seburn et Seburn 2000). Tout comme Bleakney (1958) l'avait proposé, il est possible que le climat plus clément retrouvé dans la vallée de la rivière Saguenay favorise certaines espèces de l'herpétofaune qui sont absentes du territoire environnant, lequel est plus élevé en altitude.

Il se pourrait également que la répartition de la Couleuvre à collier soit plus continue et suive une mince bande le long de la côte nord du fleuve SaintLaurent, entre la région de Québec et le Saguenay, comme pour les espèces d'arbres nommées précédemment (Farrar 1996) et conformément à la limite de la zone herpétofaunique \# 4 proposée par Bleakney (1958, page 74). Des recherches supplémentaires sont nécessaires afin de préciser la distribution de cette couleuvre au Québec, particulièrement dans le nord et l'est, notamment aux endroits isolés où les conditions sont favorables à l'espèce.

\section{Remerciements}

Les auteurs remercient Rémi Bouchard pour la découverte du spécimen, de même que Francis R. Cook, Daniel Pouliot, Benoît Couture, Hugo Royer (parc national du Saguenay) et Isabelle Picard pour leurs commentaires sur la version préliminaire du texte.

\section{Littérature citée}

Bider, J.-R., et S. Matte. 1991. Atlas des amphibiens et reptiles du Québec 1988-1989-1990, version détaillée. Société d'histoire naturelle de la vallée du Saint-Laurent et ministère du Loisir, de la Chasse et de la Pêche du Québec. Québec. 429 pages.

Bider, J.-R., et S. Matte. 1994. Atlas des amphibiens et reptiles du Québec. Société d'histoire naturelle de la vallée du Saint-Laurent et ministère de l'Environnement et de la Faune du Québec, Direction de la faune et des habitats. Québec. 106 pages.

Bleakney, J. S. 1958. A zoogeographical study of the amphibians and reptiles of Eastern Canada. National Museum of Canada, Bulletin (155), Biological Series (54). 119 pages.

Cook, F. R. 1984. Introduction aux amphibiens et reptiles du Canada. Musée national des sciences naturelles, Musées nationaux du Canada. Ottawa, Canada. 211 pages.

Desroches, J.-F., et D. Rodrigue. 2004. Amphibiens et reptiles du Québec et des Maritimes. Éditions Michel Quintin, Waterloo, Québec, 288 pages.

Ernst, C. H., et E. M. Ernst. 2003. Snakes of the United States and Canada. Smithsonian Institution, États-Unis, 668 pages.

Farrar, J. L. 1996. Les arbres du Canada. Corporation des Éditions Fides et Service canadien des forêts, Ressources naturelles Canada, Saint-Laurent, Québec, 502 pages.

MacCulloch, R. D., et J. R. Bider. 1975. New records of amphibians and Garter snakes in the James Bay area of Quebec. Canadian Field-Naturalist 89: 80-82.

Provancher, L. 1874. Faune canadienne: les reptiles. Le Naturaliste canadien 6: 353-370.

Robitaille, A., et J. - P. Saucier. 1998. Paysages régionaux du Québec méridonal. Les Publications du Québec. 213 pages + carte.

Seburn, D., et C. Seburn. 2000. Conservation priorities for the amphibians and reptiles of Canada. For World Wildlife Fund Canada and Canadian Amphibian and Reptile Conservation Network. 92 pages.

Received 27 October 2004

Accepted 24 August 2005

Revised 4 October 2005 